\title{
Precise discussion on T-asymmetry with B-meson decays
}

\author{
Hiroyuki Umeeda*, Takuya Morozumi and Hideaki Okane \\ Graduate School of Science, Hiroshima University, Higashi-Hiroshima, Hiroshima 739-8526, \\ Japan \\ Core of Research for the Energetic Universe, Hiroshima University, Higashi-Hiroshima, \\ Hiroshima 739-8526, Japan \\ E-mail: umeeda@theo.phys.sci.hiroshima-u.ac.jp, \\ morozumi@hiroshima-u.ac.jp, hideaki-ookane@hiroshima-u.ac.jp
}

\begin{abstract}
Through $B \bar{B}$ mixing system, one can construct an asymmetry which naively seems to be a time reversal (T) odd quantity. In this article, the two processes (a) $B_{-} \rightarrow \bar{B}^{0}$ and (b) $\overline{B^{0}} \rightarrow B_{-}$are used to construct the event number asymmetry. The CP violation of Kaon system denoted as $\varepsilon_{K}$ contributes to observables and we evaluate the contribution from $\varepsilon_{K}$ explicitly. The asymmetry is formulated with phase convention independent parameters which are invariant under redefinition of phase of quark fields. The overall factors of the time dependent decay rates are taken into account in this article. Furthermore, we suggest conditions for the asymmetry to be a T-odd quantity. The one of such conditions arises due to the difference of overall factors which form the asymmetry.
\end{abstract}

Flavor Physics \& CP Violation 2015

May 25-29, 2015

Nagoya, Japan

${ }^{*}$ Speaker. 


\section{Introduction}

Time reversal $(\mathrm{T})$ is a fundamental symmetry in particle physics and observation of T-asymmetry plays a crucial role to investigate the property of theoretical aspects. BaBar collaboration announced their result[1] that they observed the T-asymmetry through B-meson mixing system. The idea of taking T-asymmetry is based on Refs.[2]-[4]. BaBar stated that the observation is the first evidence of T-violation in B-system, and BaBar observable naively seems to be T-asymmetry. However, discussion in Ref.[5] shows that BaBar observable slightly deviates from a T-odd quantity.

In this article, we construct an asymmetry which consists of $B_{d}$ meson system. The difference between BaBar asymmetry and the one in this article is stated. The mixing induced $\mathrm{CP}$ violation of Kaon system contributes to the asymmetry so that one needs to take account of such mixing effect. The contribution from $\varepsilon_{K}$ to an observable is extracted. The formulation in this article is independent from phase redefinition of quark fields. For further detail, one can refer the paper[6].

\section{Time dependent asymmetry with $B_{d}$-decays}

In this section, an event number asymmetry is constructed and one can check how it behaves under T-transformation. Using processes which come from $\Upsilon(4 S)$ decay, the following processes in $B_{d}$ system are considered,

$$
\text { (a) } B_{-} \rightarrow \bar{B}^{0}, \quad \text { (b) } \bar{B}^{0} \rightarrow B_{-},
$$

where $B_{-}$indicates the $\mathrm{CP}$ odd state of $B_{d}$ meson. The processes (a) and (b) are naively related with time reversal since the time direction is opposite to each other. In Fig.1, the entire processes of (a) and (b) are shown respectively.

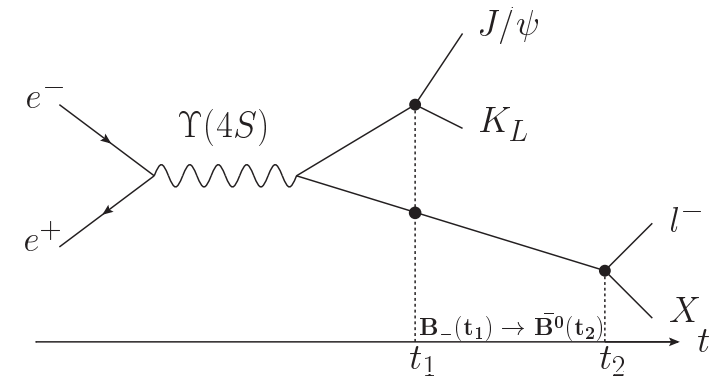

Figure 1: (Left) Process (a) in Eq.(2.1),

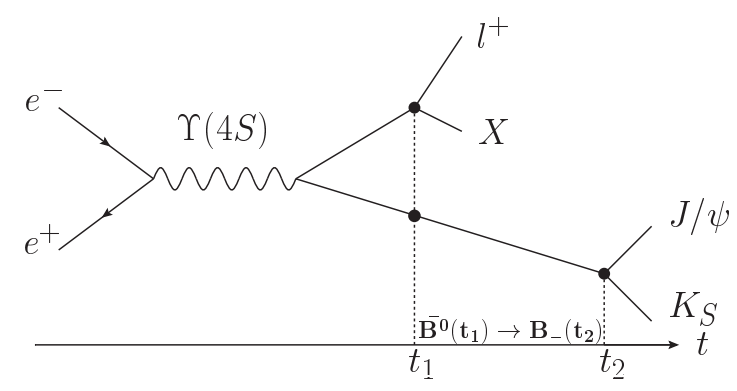

(Right) Process (b) in Eq.(2.1)

Hereafter, we explain how B mesons in (a) are identified. In (a), CP tagging is conducted at time $t_{1}$ to determine $B_{+}$(CP even B meson), and one also identify $B_{-}$due to Einstein Podolsky Rosen (EPR) correlation from $\Upsilon(4 S)$ decay. At time $t_{2}$, B meson decays into $l^{-} X$ and the flavor tagging method enables us to determine $\overline{B^{0}}$. Hence, one can select the event of $B_{-} \rightarrow \overline{B^{0}}$. Similarly, one can obtain events of (b) with flavor tagging and $\mathrm{CP}$ tagging.

Time dependent asymmetry is defined as,

$$
A(t)=\frac{\Gamma_{(a)}(t)-\Gamma_{(b)}(t)}{\Gamma_{(a)}(t)+\Gamma_{(b)}(t)},
$$


where $\Gamma_{(a)}$ and $\Gamma_{(b)}$ are time dependent decay rates for the processes (a) and (b) in Eq.(2.1). Hence, the asymmetry above is naively thought to be T-odd quantity. Here, time dependent decay rate is given as,

$$
\begin{gathered}
\Gamma_{(a)}=e^{-\Gamma\left(t_{1}+t_{2}\right)} N_{(a)} \kappa_{(a)}\left[\cosh (y \Gamma t)+\frac{\sigma_{(a)}}{\kappa_{(a)}} \sinh (y \Gamma t)+\frac{C_{(a)}}{\kappa_{(a)}} \cos (x \Gamma t)+\frac{S_{(a)}}{\kappa_{(a)}} \sin (x \Gamma t)\right], \\
t=t_{2}-t_{1}, \quad \Gamma=\frac{\Gamma_{H}+\Gamma_{L}}{2}, \quad x=\frac{m_{H}-m_{L}}{\Gamma}, \quad y=\frac{\Gamma_{H}-\Gamma_{L}}{2 \Gamma},
\end{gathered}
$$

where the coefficients $N_{(a)}, \kappa_{(a)}, \sigma_{(a)}, C_{(a)}$ and $S_{(a)}$ are given in [5][6] and we do not specify the expression of these coefficients in this article. Here the following quantities are introduced,

$$
\begin{gathered}
N_{R}=\frac{N_{(b)} \kappa_{(b)}}{N_{(a)} \kappa_{(a)}} \simeq 1+\Delta N_{R}, \\
\Delta X=\frac{1}{\sqrt{N_{R}}} \frac{X_{(a)}}{\kappa_{(a)}}-\sqrt{N_{R}} \frac{X_{(b)}}{\kappa_{(b)}}, \quad \hat{X}=\frac{1}{\sqrt{N_{R}}} \frac{X_{(a)}}{\kappa_{(a)}}+\sqrt{N_{R}} \frac{X_{(b)}}{\kappa_{(b)}}, \quad(X=\sigma, S \text { and } C)
\end{gathered}
$$

where $N_{R}$ in Eq.(2.4) is ratio of overall factors for the time dependent decay rates in Eq.(2.3). $\Delta N_{R}$ shows the effect of non-zero difference of the overall factors in Eq.(2.3). $\Delta N_{R}$ consists of small parameters such like mixing induced $\mathrm{CP}$ violation of $B_{d}$ system and CP violation in $B_{d} \rightarrow l^{-} X$ decay and $\Delta N_{R}$ is evaluated in Ref.[6]. BaBar collaboration measured the processes: $B_{-} \rightarrow \bar{B}^{0}$ and $\overline{B^{0}} \rightarrow B_{-}$. BaBar-constructed asymmetry eliminates the effect of overall factors so that the definition of BaBar asymmetry is not identical to the event number asymmetry in Eq.(2.2). In Ref.[5], BaBar asymmetry[1] is analyzed.

In this article, we investigate the event number asymmetry so that the contribution from $\Delta N_{R}$ in Eq.(2.4) needs to be taken account and the contribution from $\varepsilon_{K}$ is calculated. With the time dependent decay rate in Eq.(2.3), the asymmetry in Eq.(2.2) is given as,

$$
A(t) \simeq \frac{-\frac{\Delta N_{R}}{2}+\frac{\Delta \sigma}{2} y \Gamma t+\frac{\Delta S}{2} \sin (x \Gamma t)+\frac{\Delta C}{2} \cos (x \Gamma t)}{1+\frac{\hat{\sigma}}{2} y \Gamma t+\frac{\hat{S}}{2} \sin (x \Gamma t)+\frac{\hat{C}}{2} \cos (x \Gamma t)} .
$$

The event number asymmetry in Eq.(2.6) is expanded as,

$$
\begin{aligned}
A(t) \simeq & R_{T}+C_{T} \cos (x \Gamma t)+S_{T} \sin (x \Gamma t) \\
& +B_{T} \sin ^{2}(x \Gamma t)+D_{T} \sin (x \Gamma t) \cos (x \Gamma t)+E_{T}(y \Gamma t) \sin (x \Gamma t) .
\end{aligned}
$$

Hereafter, we discuss the coefficient of $\cos (x \Gamma t)$ and the other coefficients in Eq.(2.7) are evaluated and discussed in Ref.[6]. The following quantities are introduced,

$$
\lambda=\frac{q}{p} \frac{p_{K}}{q_{K}} \frac{\bar{A}_{\psi \bar{K}^{0}}}{A_{\psi K^{0}}} \sqrt{\frac{1+\theta_{K}}{1-\theta_{K}}}, \quad C=\frac{1-|\lambda|^{2}}{1+|\lambda|^{2}}, S=\frac{2 \operatorname{Im} \lambda}{1+|\lambda|^{2}}, \quad \theta_{K}=\frac{A_{\psi K^{0}} A_{\psi K^{0}}^{\mathrm{ID}}-\bar{A}_{\psi \bar{K}^{0}} \bar{A}_{\psi \bar{K}^{0}}^{\mathrm{ID}}}{A_{\psi K^{0}} A_{\psi K^{0}}^{\mathrm{ID}}+\bar{A}_{\psi \bar{K}^{0}} \bar{A}_{\psi \bar{K}^{0}}^{\mathrm{ID}}},
$$

where $B_{d}$ decay amplitude is denoted $A_{\psi K^{0}}$ for $B^{0} \rightarrow \psi K^{0}$ and $A_{\psi \overline{K^{0}}}$ for $B^{0} \rightarrow \psi \overline{K^{0}}$, while inverse decay amplitudes are written as $A_{\psi K^{0}}^{\mathrm{ID}}$ for $\psi K^{0} \rightarrow B^{0}$ and $A_{\psi \bar{K}^{0}}^{\mathrm{ID}}$ for $\psi \bar{K}^{0} \rightarrow B^{0}$. Mixing parameters in $B$ and $K$ systems are denoted as,

$$
\begin{gathered}
\left|K_{L}\right\rangle_{\text {in }}=p_{K} \sqrt{1+z_{K}}\left|K^{0}\right\rangle-q_{K} \sqrt{1-z_{K}}\left|\bar{K}^{0}\right\rangle,\left|K_{S}\right\rangle_{\text {in }}=p_{K} \sqrt{1-z_{K}}\left|K^{0}\right\rangle+q_{K} \sqrt{1+z_{K}}\left|\bar{K}^{0}\right\rangle \\
\left|B_{H}\right\rangle_{\text {in }}=p \sqrt{1+z}\left|B^{0}\right\rangle-q \sqrt{1-z}\left|\bar{B}^{0}\right\rangle,\left|B_{L}\right\rangle_{\text {in }}=p \sqrt{1-z}\left|B^{0}\right\rangle+q \sqrt{1+z}\left|\bar{B}^{0}\right\rangle
\end{gathered}
$$


One can check[6] that $C, S, \theta_{K}, z_{K}$ and $z$ in Eqs.(2.8-2.10) are odd or even under T-transformation. The transformation property is exhibited in Tab.1.

Table 1: Transformation properties of the parameters under T-transformation. These parameters are to express the asymmetry so that one can show the transformation property of each term.

\begin{tabular}{|c|c|c|c|c|c|c|}
\hline & $C$ & $S$ & $\theta_{K}$ & $z_{K}$ & $z$ & $\Delta \lambda_{l}$ \\
\hline $\mathrm{T}$ & - & - & + & + & + & - \\
\hline
\end{tabular}

The coefficient $C_{T}$ in Eq.(2.7) is parameterized as,

$$
C_{T}=\frac{\Delta C}{2} \simeq C-S z^{I}+\theta_{K}^{R}+S \Delta \lambda_{l}^{I} .
$$

In Eq.(2.11), $C_{T}$ is written in terms of parameters which transform odd or even under T-transformation as shown in Tab.1. $\Delta \lambda_{l}$ in Eq.(2.11) denotes wrong sign semi-leptonic decays of $B_{d}$ meson such like $B^{0} \rightarrow l^{-} X$ and $\bar{B}^{0} \rightarrow l^{+} X$. Formulation of $\Delta \lambda_{l}$ is given in Ref.[6]. One can find that the quantities in Eq.(2.11) include not only T-odd terms but also T-even contribution. This slight deviation from T-odd is pointed out in Ref.[5] regarding to BaBar asymmetry[1]. The event number asymmetry coefficients in Eq.(2.11) slightly deviate from a T-odd quantity as well as BaBar asymmetry. There exists contribution from indirect CP violation in $K$ system since the final states in Fig.1 include $K_{L}$ and $K_{S}$ which differ from exact CP eigenstates. Extracting $\varepsilon_{K}$ in Eq.(2.11), one expresses,

$$
\begin{gathered}
C_{T} \simeq C-S z^{I}+\theta_{K}^{R}+S \Delta \lambda_{l}^{I} \simeq\left(C^{\prime}-2 \operatorname{Re} \varepsilon_{K}\right)-S z^{I}+\theta_{K}^{R}+S \Delta \lambda_{l}^{I}, \\
C^{\prime}=\frac{1-\left|\lambda^{\prime}\right|^{2}}{1+\left|\lambda^{\prime}\right|^{2}}, \quad \lambda^{\prime}=\frac{q}{p} \frac{\bar{A}_{\psi \bar{K}}}{A_{\psi K^{0}}} \sqrt{\frac{1+\theta_{K}}{1-\theta_{K}}} .
\end{gathered}
$$

Under phase redefinition of a quark such like $\left\langle K^{0}\right| \rightarrow e^{-i \alpha_{K}}\left\langle K^{0}\right|$ and $\left\langle\bar{K}^{0}\right| \rightarrow e^{+i \alpha_{K}}\left\langle\bar{K}^{0}\right|, \lambda^{\prime}$ transforms as $\lambda^{\prime} \rightarrow \lambda^{\prime} e^{+2 i \alpha_{K}}$. Therefore, $C^{\prime}$ in Eq.(2.13) is shown as a phase convention independent parameter. One also sees that $\operatorname{Re} \varepsilon_{K}$ is phase convention independent and the quantities in Eq.(2.12) are phase convention independent. We find that $\operatorname{Re}_{K} \sim \mathscr{O}\left(10^{-3}\right)$ arises to contribute to $C_{T}$ in Eq.(2.12) and $C_{T}$ becomes a quantity of $\mathscr{O}\left(10^{-3}\right)$ [6].

\section{Conditions for asymmetry to be a T-odd quantity}

In the previous section, T-even parts in Eq.(2.11) are found and the coefficients slightly deviate from T-odd quantities. Hereafter, we discuss conditions for the coefficients in Eq.(2.7) to be T-odd (T-violating) quantities.

There are two types of such a condition. The first one is (1) B-meson state equivalence which is originally suggested and calculated in Ref.[5]. This condition is regarding to B-meson states which appear in Fig. 1 and it requires that B meson states should be equivalent to the states which reveal in diagrams whose time direction is totally opposite to Fig.1. (therefore such diagram contains $e^{+} e^{-}$ as a final state and $\psi K_{L} l^{-} X$ as an initial state) This is diagrammatically discussed in Ref.[6].

The second type of the conditions is (2) $\Delta N_{R}^{e}=0$; T-even part of the ratio of the overall normalizations in Eq.(2.4) should vanish. Since the event number asymmetry in Eq.(2.2) is evaluated with non-zero $\Delta N_{R}, \Delta N_{R}$ can give rise to T-even effect. This is not the case for BaBar asymmetry[1] 
since it eliminates the effect of overall normalization difference between the two processes (a) and (b) in Eq.(2.1). However, when $\Delta N_{R}^{e}=0$ is satisfied, $\Delta X(X=\sigma, C, S)$ is a T-odd quantity while $\hat{X}(X=\sigma, C, S)$ is proven[6] to be a T-even quantity. Therefore, Eq.(2.6) reads as,

$$
A(t) \simeq \frac{-\frac{\Delta N_{R}}{2}+\frac{\Delta \sigma}{2} y \Gamma t+\frac{\Delta S}{2} \sin (x \Gamma t)+\frac{\Delta C}{2} \cos (x \Gamma t)}{1+\frac{\hat{\sigma}}{2} y \Gamma t+\frac{\hat{S}}{2} \sin (x \Gamma t)+\frac{\hat{C}}{2} \cos (x \Gamma t)} \sim \frac{\mathrm{T}-\text { odd }}{\mathrm{T}-\text { even }},
$$

and $\mathrm{A}(\mathrm{t})$ turns out to be T-odd quantity. One can show that the event number asymmetry coefficients in Eq.(2.7) become T-odd when the two types of the conditions are simultaneously satisfied[6].

\section{Summary}

In this article, the event number asymmetry is investigated for the two processes: (a) $\bar{B}^{0} \rightarrow B_{-}$ and (b) $B_{-} \rightarrow \bar{B}^{0}$. The constructed asymmetry in Eq.(2.2) appears to include not only T-odd part but also T-even part, as shown in Ref.[5] with respect to BaBar asymmetry[1]. The mixing induced $\mathrm{CP}$ violation of Kaon system, i.e., $\varepsilon_{K}$ contributes to the asymmetry since the final states given in (a) and (b) contain $K_{L}$ and $K_{S}$. We extracted the effect of $\varepsilon_{K}$ and showed the contribution to an observable in Eq.(2.12). Formulation in this article is phase convention independent so that the coefficients in Eqs.(2.12) are invariant under phase redefinition of quarks.

Furthermore, we discussed the conditions for the asymmetry to be a T-odd quantity. The two types of such a condition are investigated; (1) B-meson state equivalence and (2) Vanishment of T-even part of overall normalizations' ratio of the decay rates which form the asymmetry (denoted as $\Delta N_{R}^{e}=0$ ). The condition (1) requires [5] that the B-meson state which appears in the process in Fig.1 should be equivalent to the B-meson which reveals in a process whose time direction is totally opposite to Fig.1. The condition (2) is regarding to the difference of normalization of the rates. We consider the event number asymmetry so that one should take into account the condition (2) unlike BaBar asymmetry [1]. One found that the T-even part of $\Delta N_{R}$ yields T-even contribution to the coefficients of the asymmetry. We conclude that the coefficients of the event number asymmetry become T-odd quantities when the conditions (1), (2) are simultaneously satisfied.

\section{Acknowledgments}

The authors are grateful to the FPCP2015 organizers for great opportunity of the conference. H.U. is supported by Hiroshima University Alumni Association.

\section{References}

[1] J. P. Lees et al. [BaBar Collaboration], Phys. Rev. Lett. 109, 211801 (2012) [arXiv:1207.5832].

[2] M. C. Banuls and J. Bernabeu, Phys. Lett. B 464, 117 (1999) [hep-ph/9908353].

[3] E. Alvarez and A. Szynkman, Mod. Phys. Lett. A 23, 2085 (2008) [hep-ph/0611370].

[4] J. Bernabeu, F. Martinez-Vidal and P. Villanueva-Perez, JHEP 1208, 064 (2012) [arXiv:1203.0171].

[5] E. Applebaum et al. Phys. Rev. D 89, no. 7, 076011 (2014) [arXiv:1312.4164 [hep-ph]].

[6] T. Morozumi, H. Okane and H. Umeeda, JHEP 1502, 174 (2015) [arXiv:1411.2104 [hep-ph]]. 